\title{
Life in Pause: A Time for Change (A Foot Surgeon in Greece Introspects in the Time of a Pandemic)
}

\author{
Panagiotis D Symeonidis
}

Journal of Foot and Ankle Surgery (Asia Pacific) (2020): 10.5005/jp-journals-10040-1118

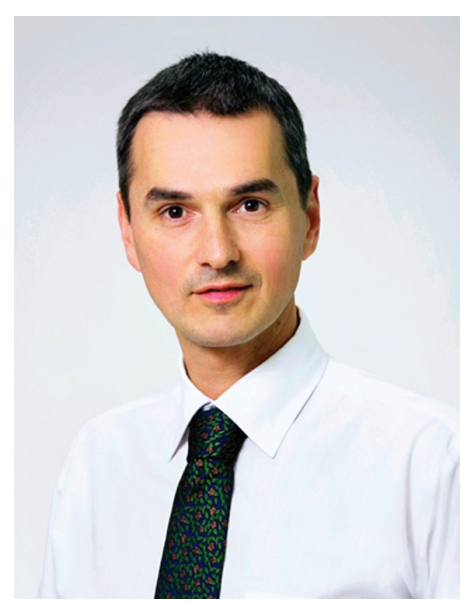

In our part of the world, the COVID-19 pandemic was not a sudden blow; rather, it was an accident waiting to happen. The gradual spread of the disease from country to country was on our daily news for months before the outbreak. The Greek government and the medical community had some time to prepare for the worst-and fortunately, they did. In proportion with other countries, Greece so far can be seen as a success story in the handling of the coronavirus. With a population of around 11 million, we experienced a relatively low death toll ( $<150$ fatalities on the date I am writing these lines), with no crowding in ICUs, and no panic and confusion in the society in general.

The Eastern Mediterranean countries share a common climate, and to a great degree a similar culture and everyday lifestyle. Why was the course of the disease in my country to this date so different in comparison to neighboring Italy? In the future, epidemiologists will be able to give scientific answers with the sobriety of evidencebased research. Still, a striking feature of Greece's strategy was that politicians listened to the medical experts, and not the other way around. From a very early stage, way before the first confirmed case, a plan was devised on how to shield our fragile public health system, which further to its inherent weaknesses was alarmingly depleted from staff and equipment after the recent financial crisis. This response I believe is similar to countries of Southeast Asia, where morbidity and mortality mirror that of Greece.

The measures were austere and absolute both in Greece and in South Asia; early and complete lockdown of schools and businesses, aggressive campaigns in all media about staying at home, social distancing, and personal hygiene seem to have been the things that worked in areas of low mortality. Cancellation of all elective surgery, consultations only for trauma and emergency, and official guidelines for patient care with strict precautions from the medical council were all enforced. Suddenly, life as we knew it came to a standstill for all of us.

As a busy foot and ankle surgeon, I found myself staying at home for a period of 6 consecutive weeks. This has been the longest time in my adult life without daily work. Patient follow-up was done over the phone and with the help of district nurses, as people were not allowed to commute unless it was an emergency. Evaluation was aided by clinical photographs, videos, e-mails and video calls, using any possible platform. In regards to trauma calls,
Orthopaedic Department, St Luke's Hospital, Thessaloniki, Greece

Corresponding Author: Panagiotis D Symeonidis, Orthopaedic Department, St Luke's Hospital, Thessaloniki, Greece, Phone: +30231025800, e-mail: p.symeonidis@gmail.com

How to cite this article: Symeonidis PD. Life in Pause: A Time for Change (A Foot Surgeon in Greece Introspects in the Time of a Pandemic). J Foot Ankle Surg (Asia Pacific) 2020;7(1):1.

Source of support: Nil

Conflict of interest: None

hospitals experienced a sudden drop of case numbers. There were far less road traffic accidents as people weren't driving. Almost no sports-related injuries apart from the odd "I suddenly decided to become fit at home" cases. No work-related trauma as construction and other businesses were shut.

Following the dreadful news from around the world on a daily basis, there was still plenty of time to rest and contemplate. The greatest hidden gift within this tragedy was the time I could spend with my family. I cherish our health and love every day and am amazed by my children's resilience and adaptation skills under these unprecedented circumstances. I am enjoying the company of my lovely wife, our long talks over cups of tea and coffee, or merely one another's silent presence.

On top of that, I find myself being very productive academically. Unexpectedly, there is time to read all these journals I only scrolled through, watch great webinars on the latest in my field, and work on a dozen of half-finished papers and projects. Finally, I had the chance to participate in a unique global webinar organized by the humanitarian organization Steps2Walk and share my passion for foot and ankle surgery with my mentors and teachers. We surgeons have realized that academic exchanges can be productive by staying at home also.

What will the next day bring? Surely there will be great pressure for treatment from patients with ongoing and deteriorating pathology and deformities. We will need to prioritize, evaluating the severity of each case and natural history of each condition. As the pandemic will be followed by a significant recession, there will be new waves of unemployment, poverty, and need. We will also need to intensify our charity work and adapt our practices, in order to meet a growing need for high-quality but low-cost patient care. What about our scientific work? Maybe we can be more creative with less noise, less unnecessary phone calls and appointments, less meaningless e-mails and social media. It will also be important to keep the momentum of this daily serenity, improving the balance between work and personal time. If there is one thing this pandemic has reminded us, even in a painful way, is that we don't need much to be content and happy in our daily life. 